\title{
Remarks on the Stability of a Functional Equation of Quadratic Type
}

\author{
By \\ Liviu Cădariu and Viorel Radu \\ (Vorgelegt in der Sitzung der math.-nat. Klasse am 11. Oktober 2007 \\ durch das w. M. Ludwig Reich)
}

\begin{abstract}
Our aim is to present some generalized stability results of Ulam-Hyers type for $\lambda$-quadratic functional equations of the form $Q_{\lambda}(F)=0$, where $\lambda \in\{1,2\}, Q_{\lambda}(F)$ is given by

$$
\begin{aligned}
Q_{\lambda}(F)(u, v):= & F(u+v)+F(u+S(v))+(\lambda-1)(F(u-v)+F(u-S(v))) \\
& -2^{\lambda}\left(F(u)+F(v)+F\left(\frac{u+S(u)+v-S(v)}{2}\right)\right. \\
& \left.+F\left(\frac{u-S(u)+v+S(v)}{2}\right)\right),
\end{aligned}
$$
\end{abstract}

and the unknown function $F$ is defined on linear spaces $Z=X_{1} \times X_{2}$ and $S=S_{X_{1}}:=$ $P_{X_{1}}-P_{X_{2}}$.

Mathematics Subject Classification (2000): 39B62, 39B72, 39B82, 47H10.

Key words: $\lambda$-Quadratic functional equation, fixed points, stability.

\section{Introduction}

Different methods to obtain stability properties for functional equations are known. The direct method revealed by HYERS in [17], where the Ulam's problem concerning the stability of homomorphisms was affirmatively answered for Banach spaces, arrived at a very large extent and successful use (see, e.g., [1], [3], [32], [16], [22]). 
The interested reader may consult [13], [18], [10], [11] and [19] for details.

On the other hand, in [27], [5] and [6] a fixed point method was proposed, by showing that many theorems concerning the stability of Cauchy and Jensen equations are consequences of the fixed point alternative. Subsequently, the method has been successfully used, e.g., in [7], [8], [31], [21], [20] or [24]. It is worth noting that the fixed point method introduces a metrical context and better clarifies the ideas of stability, which is seen to be unambiguously related to fixed points of concrete contractive-type operators on suitable (function) spaces.

We present some generalized Ulam-Hyers stability results for functional equations of $\lambda$-quadratic type. By using both the direct method and the fixed point method, we slightly extend the results in [25], [26], [9], [16], [22], [28], [29] and [30].

\section{Functional Equations of $\lambda$-Quadratic Type}

Let $X_{1}, X_{2}$ and $Y$ be real linear spaces and consider the Cartesian product $Z:=X_{1} \times X_{2}$ together with the linear selfmappings $P_{X_{1}}, P_{X_{2}}$ and $S$, where $P_{X_{1}}(u)=\left(u_{1}, 0\right), P_{X_{2}}(u)=\left(0, u_{2}\right), \forall u=\left(u_{1}, u_{2}\right) \in Z$, and $S=S_{X_{1}}:=P_{X_{1}}-P_{X_{2}}$. A function $F: Z \rightarrow Y$ is called a $\lambda$-quadratic mapping $(\lambda \in\{1,2\})$ iff it satisfies, for all $u, v \in Z$, the following equation:

$$
\begin{aligned}
Q_{\lambda}(F)(u, v):= & F(u+v)+F(u+S(v))+(\lambda-1)(F(u-v)+F(u-S(v))) \\
& -2^{\lambda}\left(F(u)+F(v)+F\left(\frac{u+S(u)+v-S(v)}{2}\right)\right. \\
& \left.+F\left(\frac{u-S(u)+v+S(v)}{2}\right)\right)=0 .
\end{aligned}
$$

Notice that, whenever $Z$ is an inner product space, $F(u)=$ $a \cdot\left\|P_{X_{1}} u\right\|^{\lambda} \cdot\left\|P_{X_{2}} u\right\|^{2}, \lambda \in\{1,2\}$, defines a solution of $(2.1)$ for each $a \in \mathbb{R}$.

For $\lambda=1$ a solution $F: Z \rightarrow Y$ is called an Add Q-type mapping. If $F$ is a solution of (2.1) for $X_{1}=X_{2}=X$, then $X \times X \ni u=$ $(x, z) \rightarrow f(x, z):=F(u) \in Y$ is an additive-quadratic mapping on $X$, i.e., it verifies the following equation [26]:

$$
\begin{aligned}
& f(x+y, z+w)+f(x+y, z-w) \\
& \quad=2(f(x, z)+f(y, w)+f(x, w)+f(y, z)), \quad \forall x, y, z, w \in X .
\end{aligned}
$$


For $\lambda=2$, a solution $F: Z \rightarrow Y$ is called a Bi Q-type mapping. If $F$ verifies (2.1) for $X_{1}=X_{2}=X$, then $u=(x, z) \rightarrow f(x, z):=F(u)$ is $a$ bi-quadratic mapping, verifying the following equation [25]:

$$
\begin{gathered}
f(x+y, z+w)+f(x+y, z-w)+f(x-y, z+w)+f(x-y, z-w) \\
=4(f(x, z)+f(y, w)+f(x, w)+f(y, z)), \quad \forall x, y, z, w \in X .
\end{gathered}
$$

Remark 2.1. Any solution $F$ of $(2.1)$ has the following properties:

(i) $F(0)=0 ; F$ is an odd mapping for $\lambda=1$ and an even mapping for $\lambda=2$;

(ii) $F\left(2^{n} \cdot u\right)=2^{(\lambda+2) n} \cdot F(u), \forall u \in Z, \forall n \in \mathbb{N}$;

(iii) $F \circ S=F$ and $F \circ P_{X_{1}}=F \circ P_{X_{2}}=0$;

(iv) moreover, if $f(x, z)=F(u)$, where $u=(x, z)$, then

(iv.1) for $\lambda=1, f$ is additive in the first variable and quadratic in the second variable;

(iv.2) for $\lambda=2, f$ is quadratic in each variable.

We also have the following

Lemma 2.1. Suppose $F: Z \rightarrow Y$ is of the form

$$
F(u)=f_{2}(z) f_{1}(x), \quad \forall u=(x, z) \in Z=X_{1} \times X_{2},
$$

with arbitrary nonzero mappings $f_{1}: X_{1} \rightarrow Y$ and $f_{2}: X_{2} \rightarrow \mathbb{R}$. Then:

(i) $F$ is 1-quadratic if $f_{1}$ is additive and $f_{2}$ is quadratic;

(ii) $f_{1}$ is additive if $F$ is 1-quadratic and $f_{2}$ is quadratic;

(iii) $f_{2}$ is quadratic if $F$ is 1-quadratic and $f_{1}$ is additive;

(iv) $F$ is 2-quadratic if and only if $f_{1}$ and $f_{2}$ are quadratic.

\subsection{The Generalized Ulam-Hyers Stability for $\lambda$-Quadratic Equations}

Let us consider a control mapping $\Phi: Z \times Z \rightarrow[0, \infty)$ such that, for all $u, v \in Z$,

$$
\begin{gathered}
\Psi(u, v):=\sum_{i=0}^{\infty} \frac{\Phi\left(2^{i} u, 2^{i} v\right)}{2^{(\lambda+2)(i+1)}}<\infty, \\
\left(\Psi(u, v):=\sum_{i=1}^{\infty} 2^{(\lambda+2)(i-1)} \Phi\left(\frac{u}{2^{i}}, \frac{v}{2^{i}}\right)<\infty, \quad \text { respectively }\right)
\end{gathered}
$$

and suppose $Y$ is a Banach space. 
Theorem 2.2. Let $F: Z \rightarrow Y$ be such a mapping that $F \circ P_{X_{1}}+$ $(\lambda-1) F \circ P_{X_{2}}=0$ and

$$
\left\|Q_{\lambda}(F)(u, v)\right\|_{Y} \leq \Phi(u, v), \quad \forall u, v \in Z .
$$

Then there exists a unique $\lambda$-quadratic mapping $B: Z \rightarrow Y$, given by $B(u)=\lim _{n \rightarrow \infty} \frac{F\left(2^{n} u\right)}{2^{(\lambda+2) n}}, \quad\left(B(u)=\lim _{n \rightarrow \infty} 2^{(\lambda+2) n} \cdot F\left(\frac{u}{2^{n}}\right)\right), \quad \forall u \in Z$, for which

$$
\|F(u)-B(u)\|_{Y} \leq \Psi(u, u), \quad \forall u \in Z .
$$

Proof. We shall use the Hyers' direct method. Letting $u=v$ in (2.5), we obtain

$$
\left\|\frac{F(2 u)}{2^{\lambda+2}}-F(u)\right\|_{Y} \leq \frac{\Phi(u, u)}{2^{\lambda+2}}, \quad \forall u \in Z .
$$

In the next step, as usual, one shows that

$$
\left\|\frac{F\left(2^{p} u\right)}{2^{(\lambda+2) p}}-\frac{F\left(2^{m} u\right)}{2^{(\lambda+2)^{m}}}\right\|_{Y} \leq \sum_{i=p}^{m-1} \frac{\Phi\left(2^{i} u, 2^{i} u\right)}{2^{(\lambda+2)(i+1)}}, \quad \forall u \in Z,
$$

for given integers $p, m$, with $0 \leq p<m$. Using (2.4) and (2.7), $\left\{F\left(2^{n} u\right) / 2^{(\lambda+2) n}\right\}_{n>0}$ is a Cauchy sequence for any $u \in Z$. Since $Y$ is complete, we can define the mapping $B: Z \rightarrow Y$,

$$
B(u)=\lim _{n \rightarrow \infty} \frac{F\left(2^{n} u\right)}{2^{(\lambda+2) n}}, \quad \forall u \in Z .
$$

By using (2.7) for $p=0$ and $m \rightarrow \infty$ we obtain the estimation (2.6).

By (2.5), we have

$$
\begin{aligned}
& \| \frac{F\left(2^{n}(u+v)\right)}{2^{(\lambda+2) n}}+\frac{F\left(2^{n}(u+S(v))\right)}{2^{(\lambda+2) n}} \\
& \quad+(\lambda-1)\left(\frac{F\left(2^{n}(u-v)\right)}{2^{(\lambda+2) n}}+\frac{F\left(2^{n}(u-S(v))\right)}{2^{(\lambda+2) n}}\right) \\
& \quad-2^{\lambda}\left(\frac{F\left(2^{n}(u)\right)}{2^{(\lambda+2) n}}+\frac{F\left(2^{n}(v)\right)}{2^{(\lambda+2) n}}+\frac{1}{2^{(\lambda+2) n}} F\left(2^{n}\left(\frac{u+S(u)+v-S(v)}{2}\right)\right)\right. \\
& \left.\quad+\frac{1}{2^{(\lambda+2) n}} F\left(2^{n}\left(\frac{u-S(u)+v+S(v)}{2}\right)\right)\right) \|_{Y} \leq \frac{\Phi\left(2^{n} u, 2^{n} v\right)}{2^{(\lambda+2) n}},
\end{aligned}
$$

for all $u, v \in Z$. Using (2.4), (2.8) and letting $n \rightarrow \infty$, we immediately see that $B$ is a $\lambda$-quadratic mapping. 
Let $B_{1}$ be a $\lambda$-quadratic mapping which satisfies (2.6). Then

$$
\begin{aligned}
\left\|B(u)-B_{1}(u)\right\|_{Y} & \leq\left\|\frac{B\left(2^{n} u\right)}{2^{(\lambda+2) n}}-\frac{F\left(2^{n} u\right)}{2^{(\lambda+2) n}}\right\|_{Y}+\left\|\frac{F\left(2^{n} u\right)}{2^{(\lambda+2) n}}-\frac{B_{1}\left(2^{n} u\right)}{2^{(\lambda+2) n}}\right\|_{Y} \\
& \leq 2 \cdot \sum_{k=n}^{\infty} \frac{\Phi\left(2^{k} u, 2^{k} u\right)}{2^{(\lambda+2)(k+1)}} \longrightarrow 0, \quad \text { for } n \rightarrow \infty .
\end{aligned}
$$

Hence the uniqueness claim for $B$ holds true.

Let us consider a mapping $\varphi: X \times X \times X \times X \rightarrow[0, \infty)$ such that $\forall x, y, z, w \in X$,

$$
\begin{gathered}
\psi(x, z, y, w):=\sum_{i=0}^{\infty} \frac{\varphi\left(2^{i} x, 2^{i} z, 2^{i} y, 2^{i} w\right)}{2^{(\lambda+2)(i+1)}}<\infty \\
\left(\psi(x, z, y, w):=\sum_{i=1}^{\infty} 2^{(\lambda+2)(i-1)} \varphi\left(\frac{x}{2^{i}}, \frac{y}{2^{i}}, \frac{z}{2^{i}}, \frac{w}{2^{i}}\right)<\infty, \text { respectively }\right) .
\end{gathered}
$$

As a direct consequence of Theorem 2.2, for $\lambda=1 / \lambda=2$, we obtain:

Corollary 2.3. Suppose that $X$ is a real linear space, $Y$ is a real Banach space and let $f: X \times X \rightarrow Y$ be a mapping such that

$$
\begin{aligned}
& \| f(x+y, z+w)+f(x+y, z-w)+(\lambda-1)(f(x-y, z+w) \\
& \quad+f(x-y, z-w))-2^{\lambda}(f(x, z)+f(y, w)+f(x, w)+f(y, z)) \|_{Y} \\
& \leq \varphi(x, z, y, w),
\end{aligned}
$$

and let $f(x, 0)+(\lambda-1) \cdot f(0, z)=0$, for all $x, y, z, w \in X$. Then there exists a unique additive-quadratic/bi-quadratic mapping $b: X \times X \rightarrow Y$, given by

$$
\begin{gathered}
b(x, z)=\lim _{n \rightarrow \infty} \frac{f\left(2^{n} x, 2^{n} z\right)}{2^{(\lambda+2) n},} \quad\left(b(x, z)=\lim _{n \rightarrow \infty} 2^{(\lambda+2) n} \cdot f\left(\frac{x}{2^{n}}, \frac{z}{2^{n}}\right)\right), \\
\forall x, z \in X,
\end{gathered}
$$

such that

$$
\|f(x, z)-b(x, z)\|_{Y} \leq \psi(x, z, x, z), \quad \forall x, z \in X .
$$

Proof. Let us consider $X_{1}=X_{2}=X, u, v \in X \times X, u=(x, z), v=$ $(y, w), F(u)=f(x, z)$, and $\Phi(u, v)=\varphi(x, z, y, w)$. Since $\Psi(u, v)=$ $\psi(x, z, y, w)<\infty$, then we can apply Theorem 2.2. Clearly, the mapping $b$, defined by $b(x, z)=B(u)$ is additive-quadratic/biquadratic and verifies (2.6). 
For $\lambda=1$ in the above Corollary, we obtain the stability result in ([26], Theorem 7) and, for $\lambda=2$, that in ([25], Theorem 7).

\subsection{Stability Results of Aoki-Rassias Type}

For particular forms of the mapping $\Phi$ in (2.4), we can obtain interesting consequences. We identify stability properties with unbounded control conditions invoking sums (AOKI [1]) and products (RASSIAS [28-30]) of powers of norms.

Let $X_{1}, X_{2}$ and $Y$ be real linear spaces. Suppose that $Z:=X_{1} \times X_{2}$ is endowed with a norm $\|u\|_{Z}$ and that $Y$ is a real Banach space.

Corollary 2.4. Let $F: Z \rightarrow Y$ be a mapping such that

$$
\left\|Q_{\lambda}(F)(u, v)\right\|_{Y} \leq \varepsilon\left(\|u\|_{Z}^{p}+\|v\|_{Z}^{q}\right), \quad \forall u, v \in Z,
$$

where $p, q \in[0, \lambda+2)$ or $p, q \in(\lambda+2, \infty)$ and $\varepsilon \geq 0$ are fixed. If $F \circ P_{X_{1}}=0$ and $(\lambda-1) F \circ P_{X_{2}}=0$, then there exists a unique $\lambda$-quadratic mapping $B: Z \rightarrow Y$, such that

$\|F(u)-B(u)\|_{Y} \leq \frac{\varepsilon}{\left|2^{\lambda+2}-2^{p}\right|} \cdot\|u\|_{Z}^{p}+\frac{\varepsilon}{\left|2^{\lambda+2}-2^{q}\right|} \cdot\|u\|_{Z}^{q}, \quad \forall u \in Z$.

Proof. Consider the mapping $\Phi: Z \times Z \rightarrow[0, \infty), \quad \Phi(u, v)=$ $\varepsilon\left(\|u\|_{Z}^{p}+\|v\|_{Z}^{q}\right)$, where $p, q \in[0, \lambda+2)$ or $p, q \in(\lambda+2, \infty)$ and $\varepsilon \geq 0$. Then (see (2.4)),

$$
\Psi(u, v)=\varepsilon \cdot \frac{\|u\|_{Z}^{p}}{\left|2^{\lambda+2}-2^{p}\right|}+\varepsilon \cdot \frac{\|v\|_{Z}^{q}}{\left|2^{\lambda+2}-2^{q}\right|}<\infty, \quad \forall u, v \in Z,
$$

and the conclusion follows directly from Theorem 2.2.

Now, suppose that $X_{1}=X_{2}=X$, where $X$ is a real normed space, and consider the function $X \times X \ni u=(x, z) \rightarrow F(u)=f(x, z)$, where $f$ is mapping $X \times X$ into the real Banach space $Y$. Although the functions of the form $u \rightarrow\|u\|:=\left(\|x\|^{r}+\|z\|^{s}\right)^{1 / t}$ may not be norms, the above proofs work as well, and we obtain the following stability properties for $\lambda$-quadratic equations:

Corollary 2.5. Let $f: X \times X \rightarrow Y$ be a mapping such that

$$
\begin{aligned}
& \| f(x+y, z+w)+f(x+y, z-w)+(\lambda-1)(f(x-y, z+w) \\
& \quad+f(x-y, z-w))-2^{\lambda}(f(x, z)+f(y, w)+f(x, w)+f(y, z)) \|_{Y} \\
& \quad \leq \varepsilon\left(\|x\|_{X}^{p}+\|y\|_{X}^{p}+\|z\|_{X}^{q}+\|w\|_{X}^{q}\right),
\end{aligned}
$$

for all $x, y, z, w \in X$ and for some fixed $\varepsilon, p, q$, with $p, q \in[0,2+\lambda)$ or $p, q \in(\lambda+2, \infty)$ and $\varepsilon \geq 0$. If $f(x, 0)=0$ and $(\lambda-1) f(0, y)=0$, 
for all $x, y \in X$, then there exists a unique additive-quadratic/biquadratic mapping $b: X \times X \rightarrow Y$, such that

$$
\|f(x, z)-b(x, z)\|_{Y} \leq \frac{2 \varepsilon}{\left|2^{\lambda+2}-2^{p}\right|} \cdot\|x\|_{X}^{p}+\frac{2 \varepsilon}{\left|2^{\lambda+2}-2^{q}\right|} \cdot\|z\|_{X}^{q}, \quad \forall x, z \in X
$$

for all $x, z \in X$.

Furthermore, by using the means inequality or directly, two interesting results of RASSIAS type can be obtained for products:

Corollary 2.6. Let $F: Z \rightarrow Y$ be a mapping such that

$$
\left\|Q_{\lambda}(F)(u, v)\right\|_{Y} \leq \varepsilon \cdot\|u\|_{Z}^{p} \cdot\|v\|_{Z}^{q}, \quad \forall u, v \in Z,
$$

where $\varepsilon, p, q \geq 0$ are fixed and $p+q \neq \lambda+2$. If $F \circ P_{X_{1}}=0$ and $(\lambda-1) F \circ P_{X_{2}}=0$, then there exists a unique $\lambda$-quadratic mapping $B: Z \rightarrow Y$, such that

$$
\|F(u)-B(u)\|_{Y} \leq \frac{\varepsilon}{\left|2^{\lambda+2}-2^{p+q}\right|} \cdot\|u\|_{Z}^{p+q}, \quad \forall u \in Z .
$$

Proof. Consider the mapping $\Phi: Z \times Z \rightarrow[0, \infty), \quad \Phi(u, v)=$ $\varepsilon \cdot\|u\|_{Z}^{p} \cdot\|v\|_{Z}^{q}$, where $\varepsilon, p, q \geq 0$ are fixed and $p+q \neq \lambda+2$. Then (see (2.4))

$$
\Psi(u, v)=\varepsilon \cdot \frac{\|u\|_{Z}^{p} \cdot\|v\|_{Z}^{q}}{\left|2^{\lambda+2}-2^{p+q}\right|}<\infty, \quad \forall u, v \in Z,
$$

so that we can apply Theorem 2.2.

Corollary 2.7. Let $f: X \times X \rightarrow Y$ be a mapping such that

$$
\begin{aligned}
& \| f(x+y, z+w)+f(x+y, z-w)+(\lambda-1)(f(x-y, z+w) \\
& \quad+f(x-y, z-w))-2^{\lambda}(f(x, z)+f(y, w)+f(x, w)+f(y, z)) \|_{Y} \\
& \leq \varepsilon \cdot\left(\|x\|_{X}^{p}+\|z\|_{X}^{p}\right) \cdot\left(\|y\|_{X}^{q}+\|w\|_{X}^{q}\right),
\end{aligned}
$$

for all $x, y, z, w \in X$ and for some fixed $\varepsilon, p, q \geq 0$, with $p+q \neq \lambda+2$. If $f(x, 0)=0$ and $(\lambda-1) f(0, y)=0$, for all $x \in X$, then there exists a unique additive-quadratic/bi-quadratic mapping $b: X \times X \rightarrow Y$, such that

$$
\begin{gathered}
\|f(x, z)-b(x, z)\|_{Y} \leq \frac{\varepsilon}{\left|2^{\lambda+2}-2^{p+q}\right|} \cdot\left(\|x\|_{X}^{p}+\|z\|_{X}^{p}\right) \cdot\left(\|x\|_{X}^{q}+\|z\|_{X}^{q}\right), \\
\forall x, z \in X .
\end{gathered}
$$




\subsection{Applications to Additive Equations and to Quadratic Equations}

A function $h: X \rightarrow Y$, between linear spaces, is called a mapping of $\lambda$-order, $\lambda \in\{1,2\}$, if it satisfies the following equation:

$$
h(x+y)+(\lambda-1) h(x-y)=2^{\lambda-1}(h(x)+h(y)), \quad \forall x, y \in X .
$$

Obviously, a mapping of 1-order is an additive mapping and a mapping of 2-order is a quadratic mapping.

For the sake of convenience, we recall the following generalized Ulam-Hyers stability properties of the additive and quadratic functional equations. Let $X$ be a real normed vector space, $Y$ a real Banach space and $\bar{\varphi}: X \times X \rightarrow[0, \infty)$ a given mapping.

$\mathbf{A}_{1}$ ([16], Theorem; see also [12]): If $\bar{\varphi}$ verifies the condition

$$
\bar{\phi}_{1}(x, y):=\sum_{i=0}^{\infty} \frac{\bar{\varphi}\left(2^{i} x, 2^{i} y\right)}{2^{i+1}}<\infty, \quad \text { for all } \quad x, y \in X
$$

and the mapping $\bar{f}: X \rightarrow Y$ satisfies the relation

$$
\|\bar{f}(x+y)-\bar{f}(x)-\bar{f}(y)\|_{Y} \leq \bar{\varphi}(x, y), \quad \text { for all } x, y \in X,
$$

then there exists a unique additive mapping $\bar{a}_{1}: X \rightarrow Y$ which satisfies the inequality

$$
\left\|\bar{f}(x)-\bar{a}_{1}(x)\right\|_{Y} \leq \bar{\phi}_{1}(x, x), \quad \text { for all } \quad x \in X .
$$

$\mathbf{A}_{2}$ ([22], Theorem 2.2): If $\bar{\varphi}$ verifies the condition

$$
\bar{\phi}_{2}(x, y):=\sum_{i=0}^{\infty} \frac{\bar{\varphi}\left(2^{i} x, 2^{i} y\right)}{2^{2(i+1)}}<\infty, \quad \text { for all } \quad x, y \in X
$$

and the mapping $\bar{f}: X \rightarrow Y$, with $\bar{f}(0)=0$, satisfies the relation

$$
\begin{gathered}
\|\bar{f}(x+y)+\bar{f}(x-y)-2 \bar{f}(x)-2 \bar{f}(y)\|_{Y} \leq \bar{\varphi}(x, y), \\
\text { for all } x, y \in X
\end{gathered}
$$

then there exists a unique quadratic mapping $\bar{a}_{2}: X \rightarrow Y$ which satisfies the inequality

$$
\left\|\bar{f}(x)-\bar{a}_{2}(x)\right\|_{Y} \leq \bar{\phi}_{2}(x, x), \quad \text { for all } \quad x \in X .
$$

As a matter of fact, we can show that the above results are consequences of our Theorem 2.2. Namely, we have 
Application 1. The stability of Eq. (2.1) implies the generalized Ulam-Hyers stability of the $\lambda$-order equation $(2.10)_{\lambda}$.

Indeed, let $X, Y, \bar{\varphi}: X \times X \rightarrow[0, \infty)$ and $\bar{f}: X \rightarrow Y$ be as in $\mathbf{A}_{\lambda}$, $\lambda \in\{1,2\}$. We take $X_{1}=X$ and consider a linear space $X_{2}$ such that there exist a quadratic function $\bar{h}: X_{2} \rightarrow \mathbb{R}$, with $\bar{h}(0)=0$ and an element $z_{0} \in X_{2}$, such that $\bar{h}\left(z_{0}\right) \neq 0$. (In inner product spaces such a function is, e.g., $z \rightarrow\|z\|^{2}$.) If we set, for $u=(x, z), v=(y, w) \in$ $X \times X_{2}$,

$$
\Phi(u, v)=\Phi(x, z, y, w)=2|\bar{h}(z)+\bar{h}(w)| \cdot \bar{\varphi}(x, y)
$$

and

$$
F(u)=F(x, z)=\bar{h}(z) \cdot \bar{f}(x),
$$

then, by using the properties of the quadratic mapping and the relations $(2.11)_{\lambda}$, for $\lambda \in\{1,2\}$, we easily get

$$
\Psi(u, v)=\frac{1}{2}|\bar{h}(z)+\bar{h}(w)| \sum_{i=0}^{\infty} \frac{\bar{\varphi}\left(2^{i} x, 2^{i} y\right)}{2^{\lambda(i+1)}}<\infty,
$$

for all $u, v \in X \times X_{2}$. At the same time, by $(2.12)_{\lambda}$,

$$
\begin{aligned}
\left\|Q_{\lambda}(F)(u, v)\right\|_{Y}= & 2|\bar{h}(z)+\bar{h}(w)| \cdot \| \bar{f}(x+y)+(\lambda-1) \bar{f}(x-y) \\
& -2^{\lambda-1}(\bar{f}(x)+\bar{f}(y)) \|_{Y} \leq 2|\bar{h}(z)+\bar{h}(w)| \cdot \bar{\varphi}(x, y) \\
= & \Phi(u, v), \quad \forall u, v \in X \times X_{2} .
\end{aligned}
$$

Therefore, by Theorem 2.2, there exists a unique mapping of $\lambda$-quadratic type, $B: X \times X_{2} \rightarrow Y$, such that $\|F(u)-B(u)\|_{Y} \leq \Psi(u, u)$ and

$$
\begin{gathered}
B(u)=\lim _{n \rightarrow \infty} \frac{F\left(2^{n} u\right)}{2^{n(\lambda+2)}}=\lim _{n \rightarrow \infty} \frac{\bar{h}\left(2^{n} z\right)}{2^{2 n}} \cdot \frac{\bar{f}\left(2^{n} x\right)}{2^{\lambda n}}=\lim _{n \rightarrow \infty} \bar{h}(z) \cdot \frac{\bar{f}\left(2^{n} x\right)}{2^{\lambda n}}, \\
\forall u=(x, z) \in X \times X_{2} .
\end{gathered}
$$

We know that $\bar{h}\left(z_{0}\right) \neq 0$. Therefore the limit

$$
\bar{a}_{\lambda}(x):=\lim _{n \rightarrow \infty} \frac{\bar{f}\left(2^{n} x\right)}{2^{\lambda n}}
$$

exists for every $x \in X$ and, moreover, $B(u)=\bar{h}(z) \cdot \bar{a}_{\lambda}(x), \forall u=$ $(x, z) \in Z$. Since $\|\bar{h}(z) \bar{f}(x)-B(u)\|_{Y} \leq \bar{h}(z) \cdot \bar{\phi}_{\lambda}(x, x), \forall u=(x, z) \in$ $X \times X_{2}$, then the estimation (2.13) ${ }_{\lambda}$ is easily seen to hold.

By Lemma 2.1, $\bar{a}_{1}$ is additive and $\bar{a}_{2}$ is quadratic. If a mapping of $\lambda$-order $\bar{c}_{\lambda}$ satisfies $(2.13)_{\lambda}$, then $(x, z) \rightarrow \vec{h}(z) \bar{c}_{\lambda}(x)$ is of $\lambda$-quadratic type (again by Lemma 2.1) and has to coincide with $B$, that is 
$\bar{h}(z) \bar{a}_{\lambda}(x)=\bar{h}(z) \bar{c}_{\lambda}(x)$, for all $u=(x, z) \in X \times X_{2}$. Since $\bar{h}$ is nonzero, then $\bar{a}_{\lambda}(x)=\bar{c}_{\lambda}(x)$, for all $x \in X$. Hence $\bar{a}_{\lambda}$ is unique.

Remark 2.2. As in the proof of Application 1 for an additive function $\bar{h}: X_{2} \rightarrow \mathbb{R}$, we can also show, by using Theorem 2.2 , that the stability of Eq. (2.1) for $\lambda=1$ implies the generalized Ulam-Hyers stability of the quadratic equation $(2.10)_{2}$.

As very particular cases, we obtain the results in AOKI [1] and RASSIAS [28] for additive equations:

Application 2. Let $\bar{f}: X \rightarrow Y$ be a mapping such that $\|\bar{f}(x+y)-\bar{f}(x)-\bar{f}(y)\|_{Y} \leq \varepsilon\left(\|x\|_{X}^{p}+\|y\|_{X}^{p}\right), \quad$ for all $\quad x, y \in X$, and for any fixed $\varepsilon, p \geq 0$, with $p \neq 1$. If $\bar{f}(0)=0$, then there exists a unique additive mapping $\bar{a}_{1}: X \rightarrow Y$ which satisfies the estimation

$$
\left\|\bar{f}(x)-\bar{a}_{1}(x)\right\|_{Y} \leq \frac{2 \varepsilon}{\left|2-2^{p}\right|} \cdot\|x\|_{X}^{p}, \quad \text { for all } \quad x \in X .
$$

Indeed, let $\bar{h}: \mathbb{R} \rightarrow \mathbb{R}, \bar{h}(z)=z^{2}$ and $\bar{f}: X \rightarrow Y$, where $X$ is a normed space and $Y$ a Banach space. We apply Theorem 2.2 for $\lambda=1$, $X_{1}=X, X_{2}=\mathbb{R}, u, v \in X \times \mathbb{R}$, with $u=(x, z), v=(y, w)$ and the mappings

$$
\begin{aligned}
F(u) & =F(x, z)=z^{2} \cdot \bar{f}(x), \\
\Phi(u, v) & =\Phi(x, z, y, w)=2\left(z^{2}+w^{2}\right) \cdot \varepsilon\left(\|x\|_{X}^{p}+\|y\|_{X}^{p}\right),
\end{aligned}
$$

to obtain the existence of a unique additive mapping $\bar{a}_{1}$ and the required estimation.

Application 3. Let $\bar{f}: X \rightarrow Y$ be a mapping such that

$\|\bar{f}(x+y)-\bar{f}(x)-\bar{f}(y)\|_{Y} \leq \theta\left(\|x\|_{X}^{p / 2} \cdot\|y\|_{X}^{p / 2}\right), \quad$ for all $\quad x, y \in X$,

and for any fixed $\theta, p \geq 0$, with $p<1$. If $\bar{f}(0)=0$, then there exists a unique additive mapping $\bar{a}_{1}: X \rightarrow Y$ which satisfies the estimation

$$
\left\|\bar{f}(x)-\bar{a}_{1}(x)\right\|_{Y} \leq \frac{\theta}{2-2^{p}} \cdot\|x\|_{X}^{p}, \quad \text { for all } x \in X .
$$

Indeed, one can use either the mappings

$$
F(u)=F(x, z)=z^{2} \cdot \bar{f}(x),
$$

and

$$
\Phi(u, v)=\Phi(x, z, y, w)=2\left(z^{2}+w^{2}\right) \cdot \theta \cdot\|x\|_{X}^{p / 2} \cdot\|y\|_{X}^{p / 2},
$$


or the means inequality:

$$
\theta\left(\|x\|_{X}^{p / 2} \cdot\|y\|_{X}^{p / 2}\right) \leq \frac{\theta}{2}\left(\|x\|_{X}^{p}+\|y\|_{X}^{p}\right)
$$

in the preceding corollary.

In particular, we obtain also a stability property of AOKI type for quadratic equations ([9]):

Application 4. Let $\bar{f}$ be a mapping from a real linear space $X$ into a real Banach space $Y$, such that

$$
\begin{aligned}
& \|\bar{f}(z+w)+\bar{f}(z-w)-2 \bar{f}(z)-2 \bar{f}(w)\|_{Y} \leq \varepsilon\left(\|z\|_{X}^{p}+\|w\|_{X}^{p}\right), \\
& \text { for all } z, w \in X,
\end{aligned}
$$

and for some fixed $\varepsilon, p \geq 0$, with $p \neq 2$. If $\bar{f}(0)=0$, then there exists a unique quadratic mapping $\bar{a}_{2}: X \rightarrow Y$ which satisfies the estimation

$$
\left\|\bar{f}(z)-\bar{a}_{2}(z)\right\|_{Y} \leq \frac{2 \varepsilon}{\left|2^{2}-2^{p}\right|} \cdot\|z\|_{X}^{p}, \quad \text { for all } \quad z \in X .
$$

For the proof, let $\bar{h}: \mathbb{R} \rightarrow \mathbb{R}, \bar{h}(x)=x$. We apply Theorem 2.2 for $\lambda=1, X_{1}=\mathbb{R}, X_{2}=X, u, v \in \mathbb{R} \times X$, with $u=(x, z), v=(y, w)$ and the mappings $F(u)=F(x, z)=x \cdot \bar{f}(z), \quad \Phi(u, v)=\Phi(x, z, y, w)=$ $|x+y| \cdot \varepsilon\left(\|z\|_{X}^{p}+\|w\|_{X}^{p}\right)$, to obtain the existence of a unique quadratic mapping $\bar{a}_{2}$ and the required estimation.

Similarly, by choosing $F(u)=F(x, z)=x \cdot \bar{f}(z)$ and $\Phi(u, v)=$ $\Phi(x, z, y, w)=|x+y| \cdot \varepsilon \cdot\|z\|_{X}^{p} \cdot\|w\|_{X}^{q}$, we obtain a stability of RASSIAS type [30]:

Application 5. Let $\bar{f}$ be a mapping from a real linear space $X$ into a real Banach space $Y$ such that $\bar{f}(0)=0$ and $\|\bar{f}(z+w)+\bar{f}(z-w)-2 \bar{f}(z)-2 \bar{f}(w)\|_{Y} \leq \varepsilon \cdot\|z\|_{X}^{p} \cdot\|w\|_{X}^{q}, \quad \forall z, w \in X$, for some fixed $\varepsilon, p, q \geq 0$, with $p+q \neq 2$. Then there exists a unique quadratic mapping $\bar{a}_{2}: X \rightarrow Y$ which satisfies the estimation

$$
\left\|\bar{f}(z)-\bar{a}_{2}(z)\right\|_{Y} \leq \frac{\varepsilon}{\left|2^{2}-2^{p+q}\right|} \cdot\|z\|_{X}^{p+q}, \quad \forall z \in X .
$$

\section{A Second Stability Result by the Fixed Point Method}

We will show that Corollary 2.4 and Corollary 2.6 can be essentially extended by using a fixed point method. The method is seen plainly related to some fixed point of a concrete operator. Specifically, our 
control conditions are perceived to be responsible for three fundamental facts: Actually, they ensure

1) the contraction property of a Schröder type operator $J$ and

2) the first two successive approximations, $f$ and $J f$, to be at a finite distance.

And, moreover, they force

3) the fixed point function of $J$ to be a solution of the initial equation.

Firstly, we prove an auxiliary result of stability for the following equation in a single variable

$$
w \circ g \circ \eta=g .
$$

Let us consider a Lipschitzian function $w: Y \rightarrow Y$, with the Lipschitz constant $L_{w}$, and the mappings $f: G \rightarrow Y, \eta: G \rightarrow G$, where $G$ is a nonempty set and $Y$ is a Banach space.

Lemma 3.1. Suppose that the mapping $f$ satisfies an inequality of the form

$$
\|(w \circ f \circ \eta)(x)-f(x)\|_{Y} \leq \psi(x), \quad \forall x \in G,
$$

where $\psi: G \rightarrow[0, \infty)$. If there exists $L<1$ such that the mapping $\psi$ has the property

$$
L_{w} \cdot(\psi \circ \eta)(x) \leq L \psi(x), \quad \forall x \in G,
$$

then there exists a unique mapping $c: G \rightarrow Y$,

$$
c(x):=\lim _{n \rightarrow \infty}\left(w^{n} \circ c \circ \eta^{n}\right)(x), \quad \forall x \in G,
$$

which satisfies the equation

$$
(w \circ c \circ \eta)(x)=c(x), \quad \forall x \in G
$$

and the inequality

$$
\|f(x)-c(x)\|_{Y} \leq \frac{\psi(x)}{1-L}, \quad \forall x \in G .
$$

Proof. Let us consider the set $\mathcal{E}:=\{g: G \rightarrow Y\}$ and introduce a complete generalized metric on $\mathcal{E}$ (as usual, inf $\emptyset=\infty$ ):

$$
d(g, h)=d_{\psi}(g, h)=\inf \left\{K \in \mathbb{R}_{+},\|g(x)-h(x)\|_{Y} \leq K \psi(x), \forall x \in G\right\} .
$$

Now, define the mapping

$$
J: \mathcal{E} \rightarrow \mathcal{E}, J g(x):=(w \circ g \circ \eta)(x) .
$$


Step I. By using the hypothesis $\left(H_{\psi}\right)$, we show that $J$ is strictly contractive on $\mathcal{E}$.

We can write, for any $g, h \in \mathcal{E}$ :

$$
d(g, h)<K \Longrightarrow\|g(x)-h(x)\|_{Y} \leq K \psi(x), \quad \forall x \in G .
$$

On the other hand,

$$
\begin{aligned}
\|J g(x)-J h(x)\|_{Y} & =\|w(g(\eta(x)))-w(h(\eta(x)))\|_{Y} \\
& \leq L_{w} \cdot\|g(\eta(x))-h(\eta(x))\|_{Y} \leq L_{w} \cdot K \cdot \psi(\eta(x)) \\
& \leq K \cdot L \cdot \psi(x), \quad \forall x \in G \Longrightarrow d(J g, J h) \leq L K .
\end{aligned}
$$

Therefore, we see that

$$
d(J g, J h) \leq L d(g, h), \quad \forall g, h \in \mathcal{E},
$$

that is $J$ is a strictly contractive self-mapping of $\mathcal{E}$, with the constant $L<1$.

Step II. Obviously, $d(f, J f)<\infty$.

In fact, by using the relation $\left(C_{\psi}\right)$, it results that $d(f, J f)<1$.

Step III. We can apply the fixed point alternative (see, e.g., [5]), and we obtain the existence of a mapping $c: G \rightarrow Y$ such that:

$-c$ is a fixed point of $J$, that is

$$
(w \circ c \circ \eta)(x)=c(x), \quad \forall x \in G .
$$

The mapping $c$ is the unique fixed point of $J$ in the set

$$
\mathcal{F}=\{g \in \mathcal{E}, d(f, g)<\infty\} .
$$

This says that $c$ is the unique mapping with both the properties (3.1) and (3.2), where

$$
\exists K \in(0, \infty) \text { such that }\|c(x)-f(x)\|_{Y} \leq K \psi(x), \quad \forall x \in G .
$$

- $d\left(J^{n} f, c\right) \underset{n \rightarrow \infty}{\longrightarrow} 0$, which implies the equality

$$
c(x):=\lim _{n \rightarrow \infty}\left(w^{n} \circ c \circ \eta^{n}\right)(x), \quad \forall x \in G .
$$

$-d(f, c) \leq \frac{1}{1-L} d(f, J f)$, which implies the inequality

$$
d(f, c) \leq \frac{1}{1-L},
$$

that is $\left(E s t_{\psi}\right)$ is seen to be true.

Let $X_{1}, X_{2}$ be linear spaces, $Z:=X_{1} \times X_{2}, Y$ a Banach space, and consider an arbitrary mapping $\Phi: Z \times Z \rightarrow[0, \infty)$. 
Theorem 3.2. Let $F: Z \rightarrow Y$ be such a mapping for which $F \circ P_{X_{1}}+$ $(\lambda-1) F \circ P_{X_{2}}=0$ and suppose that

$$
\left\|Q_{\lambda}(F)(u, v)\right\|_{Y} \leq \Phi(u, v), \quad \forall u, v \in Z .
$$

If there exists $L<1$ such that the mapping

$$
u \rightarrow \Omega(u)=\Phi\left(\frac{u}{2}, \frac{u}{2}\right)
$$

verifies the condition

$$
\Omega(u) \leq L \cdot 2^{\lambda+2} \cdot \Omega\left(\frac{u}{2}\right), \quad \forall u \in Z,
$$

and the mapping $\Phi$ has the property

$$
\lim _{n \rightarrow \infty} \frac{\Phi\left(2^{n} u, 2^{n} v\right)}{2^{(\lambda+2) n}}=0, \quad \forall u, v \in Z,
$$

then there exists a unique $\lambda$-quadratic mapping $B: Z \rightarrow Y$, such that

$$
\|F(u)-B(u)\|_{Y} \leq \frac{L}{1-L} \Omega(u), \quad \forall u \in Z .
$$

Proof. If we set $u=v$ in the relation (2.5), then we see that

$$
\left\|F(2 u)-2^{\lambda+2} F(u)\right\|_{Y} \leq \Omega(2 u), \quad \forall u \in Z .
$$

Hence

$$
\left\|\frac{F(2 u)}{2^{\lambda+2}}-F(u)\right\|_{Y} \leq \frac{\Omega(2 u)}{2^{\lambda+2}}, \quad \forall u \in Z .
$$

Now we can apply Lemma 3.1, with $w, \eta: Z \rightarrow Y, \psi: Z \rightarrow[0, \infty)$,

$$
w(u):=\frac{u}{2^{\lambda+2}}, \quad \eta(u):=2 u, \quad \psi(u):=\frac{\Omega(2 u)}{2^{\lambda+2}} .
$$

Clearly, $L_{w}=1 / 2^{\lambda+2}$ and, by using (3.4) and the hypothesis $\left(H_{\lambda}\right)$, we obtain that $\left(C_{\psi}\right)$ and $\left(H_{\psi}\right.$ ) hold.

Then there exists a unique mapping $B: Z \rightarrow Y$,

$$
B(u):=\lim _{n \rightarrow \infty}\left(w^{n} \circ B \circ \eta^{n}\right)(u)=\lim _{n \rightarrow \infty} \frac{F\left(2^{n} u\right)}{2^{(\lambda+2) n}}, \quad \forall u \in Z,
$$

which satisfies the following equation

$$
(w \circ B \circ \eta)(u)=B(u) \Leftrightarrow B(2 u)=2^{\lambda+2} B(u), \quad \forall u \in Z
$$


and the inequality

$\|F(u)-B(u)\|_{Y} \leq \frac{\psi(u)}{1-L}=\frac{\Omega(2 u)}{2^{\lambda+2}} \cdot \frac{1}{1-L} \leq \Omega(u) \frac{L}{1-L}, \quad \forall u \in Z$.

The statement that $B$ is a $\lambda$-quadratic mapping is easily seen: If we replace $u$ by $2^{n} u$ and $v$ by $2^{n} v$ in (2.5), then we obtain

$$
\begin{aligned}
& \| \frac{F\left(2^{n}(u+v)\right)}{2^{(\lambda+2) n}}+\frac{F\left(2^{n}(u+S(v))\right)}{2^{(\lambda+2) n}} \\
& \quad+(\lambda-1)\left(\frac{F\left(2^{n}(u-v)\right)}{2^{(\lambda+2) n}}+\frac{F\left(2^{n}(u-S(v))\right)}{2^{(\lambda+2) n}}\right)-2^{\lambda}\left(\frac{F\left(2^{n}(u)\right)}{2^{(\lambda+2) n}}\right. \\
& \quad+\frac{F\left(2^{n}(v)\right)}{2^{(\lambda+2) n}}+\frac{1}{2^{(\lambda+2) n}} F\left(2^{n}\left(\frac{u+S(u)+v-S(v)}{2}\right)\right) \\
& \left.\quad+\frac{1}{2^{(\lambda+2) n}} F\left(2^{n}\left(\frac{u-S(u)+v+S(v)}{2}\right)\right)\right) \|_{Y} \leq \frac{\Phi\left(2^{n} u, 2^{n} v\right)}{2^{(\lambda+2) n}}
\end{aligned}
$$

for all $u, v \in Z$. By using (3.5) and $\left(H_{\lambda}^{*}\right)$ and letting $n \rightarrow \infty$, we see that $B$ satisfies (2.1).

Example 3.1. If we apply Theorem 3.2 with the mappings $\Phi: Z \times Z \rightarrow$ $[0, \infty)$ given by $(u, v) \rightarrow \varepsilon\left(\|u\|_{Z}^{p}+\|v\|_{Z}^{q}\right)$ and $(u, v) \rightarrow \varepsilon\|u\|_{Z}^{p} \cdot\|v\|_{Z}^{q}$, then we obtain the stability results in Corollary 2.4 and Corollary 2.6, respectively.

As it is well known (see $[15,18,9]$ ), GAJDA/CZERWIK showed that the additive/quadratic equation $(2.12)_{\lambda}$ is not stable for $\bar{\varphi}(x, y)$ of the form $\varepsilon\left(\|x\|^{\lambda}+\|y\|^{\lambda}\right), \varepsilon$ being a given positive constant $(\lambda \in\{1,2\})$. In fact, it has been proved that there exists a mapping $\bar{f}_{\lambda}: \mathbb{R} \rightarrow \mathbb{R}$ such that $(2.12)_{\lambda}$ holds with the above $\bar{\varphi}$, and there exists $n o$ additive/quadratic mapping $\bar{a}$ to verify

$$
\left|\bar{f}_{\lambda}(x)-\bar{a}_{\lambda}(x)\right| \leq c(\varepsilon)|x|^{\lambda}, \quad \text { for all } \quad x \in \mathbb{R} .
$$

This suggests the following

Example 3.2. Let $X_{1}=X_{2}=Y=\mathbb{R}$, with the Euclidean norm, and $\bar{h}: \mathbb{R} \rightarrow \mathbb{R}$ a quadratic function with $\bar{h}(0)=0, \bar{h}(1)=1$. Then $E q$. (2.1) is not stable for

$$
\Phi(u, v)=\Phi(x, z, y, w)=2 \varepsilon \cdot\left(|x|^{\lambda}+|y|^{\lambda}\right)(\bar{h}(z)+\bar{h}(w)) .
$$

In fact, we can show that there exists an $F$ for which the relation (2.5) holds and there exists no Add $Q / B i Q$-type mapping $B: X_{1} \times X_{2} \rightarrow Y$ 
to verify

$$
|F(u)-B(u)| \leq c(\varepsilon) \bar{h}(z)|x|^{\lambda}, \quad \forall u=(x, z) \in X_{1} \times X_{2} .
$$

Indeed, for $F(u)=F(x, z)=\bar{h}(z) \cdot \bar{f}_{\lambda}(x)$, and $\Phi$ as in (3.6), (2.5) holds. Therefore

$$
\begin{gathered}
\left|f(x+y)+(\lambda-1) f(x-y)-2^{\lambda-1}(f(x)+h(y))\right| \leq \varepsilon\left(|x|^{\lambda}+|y|^{\lambda}\right), \\
\text { for all } x, y \in X_{1} .
\end{gathered}
$$

Let us suppose, for a contradiction, that there exists an Add $Q / B i Q$ type mapping $B$ which verifies (3.7). By Remark 2.1, the mapping $\bar{a}_{\lambda}: X_{1} \rightarrow Y, \bar{a}_{\lambda}(x):=B(x, 1)$ is a solution for $(2.10)_{\lambda}$. The estimation (3.7) gives us

$$
\left|\bar{f}_{\lambda}(x)-\bar{a}_{\lambda}(x)\right| \leq c(\varepsilon)|x|^{\lambda}, \quad \forall x \in X_{1},
$$

in contradiction with the above result of GAJDA/CZERWIK.

\section{References}

[1] AOKI, T. (1950) On the stability of the linear transformation in Banach spaces. J. Math. Soc. Japan 2: 64-66

[2] BAKER, J. A. (1991) The stability of certain functional equations. Proc. Amer. Math. Soc. 3: 729-732

[3] Bourgin, D. G. (1951) Classes of transformations and bordering transformations. Bull. Amer. Math. Soc. 57: 223-237

[4] CĂDARIU, L. (2002) A general theorem of stability for the Cauchy's equation. Bull. Şt. Univ. Politehnica Timişoara, Seria Matematică-Fizică 47(61) (no. 2): $14-28$

[5] CĂDARIU, L., RAdU, V. (2003) Fixed points and the stability of Jensen's functional equation. J. Inequal. Pure and Appl. Math. 4(1): Art. 4 (http:// jipam.vu.edu.au)

[6] CĂDARIU, L., RADU, V. (2004) On the stability of Cauchy's functional equation: A fixed points approach. In: Sousa Ramos, J., Gronau, D., Mira, C., ReICH, L., Sharkovsky, A. (eds.) Iteration Theory (ECIT '02), Proceedings of the European Conference of Iteration Theory, Evora, Portugal, September 1-7, 2002, Grazer Math. Ber. 346: 43-52

[7] CĂDARIU, L. (2005) Fixed points in generalized metric spaces and the stability of a quartic functional equation. Bull. Şt. Univ. "Politehnica" Timişoara, Seria Matematică-Fizică 50(64) (no. 2): 25-34

[8] CĂDARIU, L., RADU, V. (2007) Fixed points in generalized metric spaces and the stability of a cubic functional equation. In: CHO, Y. J., KIM, J. K., KANG, S. M. (eds.) Fixed Point Theory and Applications, Vol. 7, pp. 53-68. Nova Science Publ., Hauppauge, NY

[9] CZERWIK, S. (1992) On the stability of quadratic mapping in normed spaces. Abh. Math. Sem. Univ. Hamburg 62: 59-64 
[10] CzERwIK, S. (2002) Functional Equations and Inequalities in Several Variables. World Scientific, Singapore

[11] DaroczY, Z., PALEs, Zs. (eds.) (2002) Functional Equations - Results and Advances. Kluwer, Dordrecht

[12] FORTI, G. L. (1980) An existence and stability theorem for a class of functional equations. Stochastica 4: 23-30

[13] FORTI, G. L. (1995) Hyers-Ulam stability of functional equations in several variables. Aequationes Math. 50: 143-190

[14] FORTI, G. L. (2004) Comments on the core of the direct method for proving Hyers-Ulam stability of functional equations. J. Math. Anal. Appl. 295(1): $127-133$

[15] GAJDA, Z. (1991) On stability of additive mappings. Internat. J. Math. Math. Sci. 14: $431-434$

[16] GĂVRUŢA, P. (1994) A generalization of the Hyers-Ulam-Rassias stability of approximately additive mappings. J. Math. Anal. Appl. 184: 431-436

[17] HYERS, D. H. (1941) On the stability of the linear functional equation. Proc. Natl. Acad. Sci. USA 27: 222-224

[18] Hyers, D. H., IsAC, G., RASSIAS, Th. M. (1998) Stability of Functional Equations in Several Variables. Birkhäuser, Basel

[19] JuNG, S. M. (2002) Hyers-Ulam-Rassias Stability of Functional Equations in Mathematical Analysis. Hadronic Press, Palm Harbor, FL

[20] JUNG, S.-M., KIM, T.-S. (2006) A fixed point approach to the stability of cubic functional equations. Bol. Soc. Mat. Mexicana 3(12): 51-57

[21] JunG, S.-M. (2007) A fixed point approach to the stability of isometries. J. Math. Anal. Appl. 329: 879-890

[22] KIM, G. H. (2001) On the stability of the quadratic mapping in normed spaces. Internat. J. Math. Math. Sci. 25(4): 217-229

[23] Margolis, B., DiAZ, J. B. (1968) A fixed point theorem of the alternative for contractions on a generalized complete metric space. Bull. Amer. Math. Soc. 74: 305-309

[24] MiRzaVAziri, M., Moslehian, M. S. (2006) A fixed point approach to stability of a quadratic equation. Bull. Braz. Math. Soc. 37 (no. 3): 361-376

[25] PARK, W. G., BAE, J. H. (2005) On a bi-quadratic functional equation and its stability. Nonlinear Analysis 62(4): 643-654

[26] PARK, W. G., BAE, J. H., Chung, B.-H. (2005) On an additive-quadratic functional equation and its stability. J. Appl. Math. Comp. 18(1-2): 563-572

[27] RADU, V. (2003) The fixed point alternative and the stability of functional equations. Fixed Point Theory 4 (no. 1): 91-96

[28] RASSIAS, J. M. (1982) On approximation of approximately linear mappings by linear mappings. J. Funct. Anal. 46 (no. 1): 126-130

[29] RASSIAS, J. M. (1989) Solution of a problem of Ulam. J. Approx. Theory 57 (no. 3): 268-273

[30] RASSIAS, J. M. (1992) On the stability of the Euler-Lagrange functional equation. C. R. Acad. Bulgare Sci. 45 (no. 6): 17-20

[31] RASsias, J. M. (2006) Alternative contraction principle and alternative Jensen and Jensen type mappings. Internat. Journal of Applied Math. \& Stat. 4 (no. M06): 1-10

[32] RASSIAS, TH. M. (1978) On the stability of the linear mapping in Banach spaces. Proc. Amer. Math. Soc. 72: 297-300 
32 L. Cădariu and V. Radu: Stability Results for a Quadratic Equations

[33] Rus, I. A. (2001) Generalized Contractions and Applications. Cluj University Press, Cluj-Napoca

[34] Ulam, S. M. (1960) A Collection of Mathematical Problems. Interscience, New York; Ulam, S. M. (1964) Problems in Modern Mathematics. Wiley, New York

Authors' addresses: Dr. Liviu Cădariu, Departamentul de Matematică, Universitatea Politehnica din Timişoara, Piaţa Victoriei 2, 300006 Timişoara, România. E-Mail: liviu.cadariu@mat.upt.ro, lcadariu@yahoo.com; Dr. Viorel Radu, Facultatea de Matematică, Universitatea de Vest din Timişoara, Bv. Vasile Pârvan 4, 300223 Timişoara, România. E-Mail: radu@math.uvt.ro. 